\title{
Frequency-Based Load Control in Power Systems
}

\author{
Changhong Zhao*, Ufuk Topcu* and Steven H. Low* \\ ${ }^{*}$ Division of Engineering and Applied Science \\ California Institute of Technology, Pasadena, California 91125
}

\begin{abstract}
Maintaining demand-supply balance and regulating frequency are key issues in power system control. Conventional approaches focus on adjusting the generation so that it follows the load. However, relying on solely regulating generation is inefficient, especially for power systems where contingencies like sudden loss in generation or sudden change in load frequently occur and the proportion of intermittent renewable power is increasing. We present a frequency-based load control scheme for demand-supply balancing and frequency regulation. We formulate a load control optimization problem which aims to balance the change in load with the change in supply while minimizing the overall end-use disutility. By studying the power system model that characterizes the frequency response to real power imbalance between demand and supply, we design decentralized synchronous and asynchronous algorithms which take advantage of local frequency measurements to solve the load control problem. Case studies show that the proposed load control scheme is capable of relatively quickly balancing the power and restoring the frequency under generation-loss like contingencies or renewable power penetration. Case studies also show that the proposed scheme still works well when users have the knowledge of a simplified system model instead of an accurate one.
\end{abstract}

\section{INTRODUCTION}

In power systems, the imbalance between generation and load must get corrected within short periods, otherwise it will drive the power line frequency to deviate from the nominal value (e.g., $60 \mathrm{~Hz}$ ). Large frequency deviation may threaten the stability and security of the power system, or even worse, cause permanent damage to the facilities [1][2]. Hence balancing generation with load and regulating frequency at the normal level has been a significant topic in power system operations.

Multiple control approaches have been applied to serve these goals. Conventional control efforts focus on the generation side. For example, the automated generation control (AGC) issues signals to control the reserved generation capacity and minimize the area control error (ACE), which includes both frequency deviation and unscheduled tie-line power flows [3][4]. The spinning reserve is a set of frequency responsive generators that automatically increase their output when a sudden loss of supply occurs [3]. In addition, many generators are equipped with speed governors which adjust the mechanical power proportionally to the frequency deviation [5][7]. Other control components include the exciter and the power system stabilizer (PSS) [8][9].

Generation side control, though widely used, has many inadeqancies. For example, the AGC takes as long as 5-15 minutes to drive the ACE to its setpoint [4]. The spinning reserve generators cannot immediately respond to a contigency, and they must be grid connected and operating in a part-loaded state, thus increasing the cost and emissions [3]. The speed governor typically has a droop characteristic so that it cannot eliminate the steady state frequency deviation [8]. Today, purely relying on the generation control is not only prohibitively expensive but also technically difficult [10]. As a complementary, a lot of works have investigated the potential of load participation in power system control, among which the load control based on local frequency measurement has been highlighted.

Loads are typically controlled via load-shedding, where a whole region of loads are disconnected from the grid by a underfrequency load-shedding (UFLS) relay as a last resort to avoid system failure [2][12]. However, additional granularity can be achieved by individual loads to be controlled. Loads with energy storage, such as heaters, air conditioners, refrigerators and PEVs, can be modulated with reasonable disturbance to customer comforts [11], so they are good candidates for load control. Equipped with frequency sensors, they can sense the frequency as a measure of supply-demand imbalance, and respond in less than 1 second [13].

Schweppe et al. presented the idea of individual load control through responding to frequency as early as 1979 [14][15]. Taylor et al. developed a distributed fuzzy load controller for renewable energy systems, which uses both the frequency and the rate of change of frequency to minimize the frequency deviations [16][17]. Trudnowski et al. assumed the loads can be adjusted proportionally to frequency deviations, and investigated multiple issues such as the distribution of loads, time delay and discretized load action [5][6]. Molina-Garcia et al. studied the aggregated load response characteristics when each load is turned on and off as the frequency goes across some thresholds [2]. The Grid Friendly Appliance controller [18] developed by the Pacific Northwest National Laboratory suggests that individual appliances can provide fast reserve within seconds by responding to certain trends in the frequency.

All the works above show the advantage of frequencybased load control that communication between loads and a central controller is no longer essential and the control is decentralized, since each load can measure the frequency signal locally. However, these works are mostly based on experiments, and do not prove convergence of their decentralized schemes. Moreover, they do not consider the user acceptance as a system-level requirement. An acceptable control strategy should affect end-use functions as little as possible, otherwise users may withdraw from the control program and the reserve capacity may decrease [3]. 
In this paper, we maintain the advantage that local frequency measurements decentralize the load control. In addition, we address the user acceptance issue by formulating an load control optimization problem whose objective is to minimize the overall end-use disutility and whose constraint is the demand-supply power balance. To solve the problem, we develop a synchronous algorithm which assumes all loads sense the frequency without measurement delays and make decisions synchronously, and then consider a more practical asynchronous algorithm which incorporates frequency measurement delay and asynchronous decision making. We prove the convergence of the proposed algorithms, with which the load control problem can be solved in a decentralized way. Besides, we discuss the effect of using a simplified system model instead of an accurate one, since in practice users may not get the exact knowledge of the system model and a simplified model can make modeling and computation easier. Numerical experiments show that the proposed load control scheme is able to drive demand-supply imbalance and frequency deviation to zero within 10 to 20 seconds after a sudden generation loss, and is relatively effective in regulating frequency and maintaining demand-supply balance under fluctuating wind generation. Moreover, the proposed scheme works well when a simplified system model, instead of an accurate one, is used.

This paper is organized as follows. Section II describes the system model and sets up the load control optimization problem. Section III presents the load control algorithms and proves their convergence. Section IV shows the case studies, including system settings and the results of numerical experiments. Section V discusses the limitations in the proposed scheme, and suggests several possible directions for future work. Finally, Section VI concludes the paper.

\section{MODEL AND PROBLEM SETUP}

In this section we introduce the power system model upon which the frequency-based load control scheme is built. Then we formulate an optimization problem that describes two goals of load control: minimizing the overall end-use disutility caused by change in load, and balancing demand with supply.

\section{A. System model}

Figure 1 shows the schematic diagram of the power system under consideration. On the generator side, multiple control approaches regulate the magnitude of stator voltage $\left|E_{a}^{\prime}\right|$, the rotor angle $\delta$, the frequency $\omega=d \delta / d t$ and the terminal voltage $V_{a}$. For example, the exciter adjusts the field-winding voltage $E_{f d}$ based on the measurement of $V_{a}$; the speed governor adjusts the mechanical power $P_{m}$ based on the measurement of $\omega$. Electrical power $P_{e}$ is supplied to the load bus via the transmission line. Let $I=\{1,2, \ldots N\}$ denote the set of loads or users (we use these two terms interchangeably). Each load $i \in I$ consumes real power $d_{i}$ which is regulated by a controller $L C_{i} . \Delta g$ denotes a real power disturbance which causes demand-supply imbalance and frequency deviation. For example, $\Delta g$ may be unscheduled power flow from another

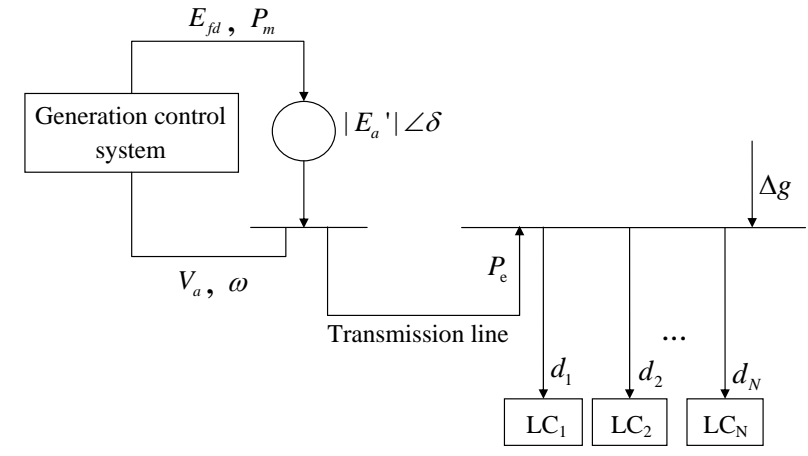

Fig. 1. Schematic diagram of the system under consideration. $\left|E_{a}^{\prime}\right|$ is the magnitude of generator stator voltage, $\delta$ is the rotor angle. The generation control system measures the terminal voltage $V_{a}$ and frequency $\omega$, and adjusts the field-winding voltage $E_{f d}$ and the turbine mechanical power $P_{m} . P_{e}$ is the power supplied to the loads. Each load $i \in\{1, \ldots, N\}$ is equipped with a load controller $L C_{i}$ that modulates load power $d_{i}$ when a power disturbance $\Delta g$ occurs.

bus, power supplied by a renewable generation source, a sudden load change, or a sudden generation loss. The case $\Delta g>0$ may stand for the increase of supply or the decrease of load.

For each $i \in I, L C_{i}$ measures the frequency deviation from the nominal value (e.g., $60 \mathrm{~Hz}$ ), $\Delta \omega$, and calculates the change in load $\Delta d_{i}$. Let $\Delta d_{i}>0$ stand for decreasing the load power, then the total load change is $\Delta d=-\sum_{i \in I} \Delta d_{i}$. Define $u:=\Delta d-\Delta g$, which is actually the demandsupply power imbalance. Then we have a system process that characterizes the relation between frequency deviation $\Delta \omega$ and power imbalance $u$. We linearize the process around some operating point, get a linear model $M$ and describe $M$ by a transfer function $G(s)$ such that $\Delta \omega(s)=G(s) u(s)$, as is a usual approach to simplify the power system analysis [22][23]. Note that $M$ is continuous-time, while the load control system is a sampling control system, i.e., users sample $\Delta \omega$ and make decisions once every $\Delta t$ time, and hold their decisions within the sampling interval. We use $t \in T=\{1,2, \ldots\}$ instead of $t=\Delta t, 2 \Delta t, \ldots$ to denote the time. Including the samplers and holders in $M$, we have a discrete-time realization, denoted by $\{A, B, C\}$, such that

$$
\begin{aligned}
x_{t+1} & =A x_{t}+B u_{t} \\
\Delta \omega_{t} & =C x_{t},
\end{aligned}
$$

where $x \in \mathbb{R}^{n}$ is the state vector, $A \in \mathbb{R}^{n \times n}, B \in \mathbb{R}^{n \times 1}$, and $C \in \mathbb{R}^{1 \times n}$

Note that $\{A, B, C\}$ exists if the transfer function $G(s)$ has a continuous-time realization (at least one exists, e.g. in the canonical form [28]), denoted by $\left\{A_{c}, B_{c}, C_{c}\right\}$, such that

$$
\begin{aligned}
\dot{x}(t) & =A_{c} x(t)+B_{c} u(t) \\
\Delta \omega(t) & =C_{c} x(t),
\end{aligned}
$$

and $A_{c}$ is non-singular with all the eigenvalues having negative real parts. To see this, note that $u$ is held constant within the 
sampling interval, so

$$
\begin{aligned}
& A=e^{A_{c} \Delta t}, \\
& B=A_{c}^{-1}\left(A-I_{n}\right) B_{c}, \\
& C=C_{c} .
\end{aligned}
$$

Moreover, in the load control scheme proposed later, we require the condition $C B \neq 0$ (see (10)), which holds for the models we use as a partial justification. Hence we make the following assumption.

Assumption 1. There exists a realization $\{A, B, C\}$ of the system process model $M$ such that $C B \neq 0$.

With the model introduced above, we now formulate an optimization problem that captures the goals of our load control scheme.

\section{B. Load control optimization problem}

The goal of the load control scheme proposed here is to balance power demand and supply, i.e., to drive $u$ to zero. By $\Delta \omega(s)=G(s) u(s)$, the frequency deviation will be driven to zero if $u$ is zero. The proposed control scheme simultaneously takes end-use functions as a system-level consideration, by minimizing the overall disutility caused by changing the loads.

We consider a constant $\Delta g$, and without loss of generality, assume $\Delta g<0$. Each user $i \in I$ has a disutility value $D_{i}\left(\Delta d_{i}\right)$ which is a function of $\Delta d_{i} \in\left[0, \bar{d}_{i}\right]$, where $\bar{d}_{i}$ is the maximum change in load $i$ allowed by appliance design or user permission. Considering the goal of minimizing overall disutility and making $u=0$, given the disturbance $\Delta g$ and the set of users $I$, we formulate the following load control problem, denoted by $\mathbf{P P}$.

$$
\mathbf{P P} \begin{cases}\min _{\Delta d_{i} \in\left[0, \bar{d}_{i}\right]} & \sum_{i=1}^{N} D_{i}\left(\Delta d_{i}\right) \\ \text { subject to } & -\sum_{i=1}^{N} \Delta d_{i}-\Delta g=0 .\end{cases}
$$

For feasibility of $\mathbf{P P}$, we need $-\sum_{i \in I} \bar{d}_{i}<\Delta g$, i.e., the magnitude of disturbance does not exceed the capability of changing the loads, which is true if enough loads participate in the load control scheme. We make the following two assumptions on the disutility functions $D_{i}$.

Assumption 2. For $i \in I, D_{i}$ is increasing, strictly convex and twice continuously differentiable over $\left[0, \bar{d}_{i}\right]$.

Assumption 3. For $i \in I$, there exists $\bar{\alpha}_{i}>0$ so that $D_{i}^{\prime \prime}\left(\Delta d_{i}\right) \geq 1 / \bar{\alpha}_{i}$ for $\Delta d_{i} \in\left[0, \bar{d}_{i}\right]$.

With Assumptions 2-3, PP becomes a convex problem which can be solved using the decentralized approach proposed later. Moreover, the reasonability of Assumptions 2-3 can be implied from previous works on demand response, where the utility functions are non-decreasing and concave, which means users are interested to consume more power if possible, and the level of satisfaction for users can gradually get saturated. For example, the utility functions often take the quadratic form and have linear marginal benefits [30][31].
Solving PP by a central controller requires the knowledge of $D_{i}$ for all $i \in I$. However, for privacy considerations, users may not prefer to reveal their disutility functions to the utility company or any other organization that controls the system. Moreover, the disutility functions may take a complex form and change with time, so it would cost much to communicate the disutility fucntions to a central controller. Therefore, we propose a decentralized approach to solve PP. In order to derive this decentralized procedure, we consider the dual problem of PP. Define the Lagrangian of PP

$$
L\left(\Delta d_{i}, p\right):=\sum_{i=1}^{N} D_{i}\left(\Delta d_{i}\right)+p\left(-\sum_{i=1}^{N} \Delta d_{i}-\Delta g\right) .
$$

Then, the dual objective function is

$$
\begin{aligned}
U(p) & =\inf _{\Delta d_{i} \in\left[0, \bar{d}_{i}\right]} L\left(\Delta d_{i}, p\right) \\
& =\sum_{i=1}^{N} \min _{\Delta d_{i} \in\left[0, \bar{d}_{i}\right]}\left(D_{i}\left(\Delta d_{i}\right)-p \Delta d_{i}\right)-p \Delta g .
\end{aligned}
$$

Hence, we have the dual problem, denoted by DP.

$$
\mathbf{D P}\left\{\max _{p} U(p) .\right.
$$

The solution to $\mathbf{P P}$ can be constructed in a decentralized way, from DP. To see this, note that given $p \in \mathbb{R}$, the problem

$$
\min _{\Delta d_{i} \in\left[0, \bar{d}_{i}\right]} D_{i}\left(\Delta d_{i}\right)-p \Delta d_{i}
$$

has a unique minimizer denoted by

$$
\Delta d_{i}(p)=\min \left\{\max \left\{\left(D_{i}^{\prime}\right)^{-1}(p), 0\right\}, \bar{d}_{i}\right\},
$$

since $D_{i}$ is strictly convex. Note that the inverse of $D_{i}^{\prime}$ exists over $\left[D_{i}^{\prime}(0), D_{i}^{\prime}\left(\bar{d}_{i}\right)\right]$ since $D_{i}^{\prime}$ is continuous and strictly increasing by Assumption 2. Since $D_{i}$ is convex for $i \in I$ and $\mathbf{P P}$ has affine constraints, Slater's condition implies that there is zero duality gap between $\mathbf{P P}$ and $\mathbf{D P}$, and the dual optimal solution, denoted by $p^{*}$, is attained [26, Sec. 5.5.3]. It follows that $d\left(p^{*}\right):=\left[d_{1}\left(p^{*}\right), \ldots, d_{N}\left(p^{*}\right)\right]^{T}$ is primal feasible and optimal [26, Sec. 5.5.2]. Therefore, we can focus on solving the dual problem DP to solve PP, instead of solving PP directly.

\section{FREQUENCY-BASED LOAD CONTROL ALGORITHMS}

In this section, we first introduce an input reconstructor which forms the basis of the frequency-based load control algorithms by reconstructing the input $u$ to the system (which is actually the gradient value of the dual objective function) locally at each load and enabling solving DP with a decentralized gradient method. Then we introduce the algorithms, including a synchronous version and an asynchronous version. In the algorithms, each user solves its local problem, so that the changes in the loads converge to the solution of the load control problem PP. 


\section{A. Input reconstructor}

As introduced in Section II-B, given the maximizer of DP, denoted by $p^{*}$, the optimal change in load $i$ is $\Delta d_{i}\left(p^{*}\right)$. On the other hand, we can get $p^{*}$ using the gradient algorithm [20, Sec. 3.2.1], where at each time $t$ the value of $p$ is adjusted in the direction of the gradient $U^{\prime}(p)$ as

$$
p(t+1)=p(t)+\gamma U^{\prime}(p(t)),
$$

where $\gamma>0$ is a stepsize. Recall that $\Delta d_{i}(p)$ denotes the unique minimizer of (4) for given $p$. By (3), if the change in load $i$ at time $t$ is $\Delta d_{i}(t)=\Delta d_{i}(p(t)),{ }^{1}$ we have

$$
U^{\prime}(p(t))=-\sum_{i=1}^{N} \Delta d_{i}(t)-\Delta g=u_{t},
$$

where $u_{t}$ denotes the value of demand-supply imbalance $u$ at time $t$. Substituting (7) into (6) we have

$$
p(t+1)=p(t)+\gamma u_{t} .
$$

Therefore, the key to decentralizing the load control is to reconstruct the value of $u$ locally at each load. Since $u$ is the input to the power system modeled by $M$ (see Section II-A), we call the mechanism of reconstructing $u$ as the input reconstructor. In the frequency-based load control scheme, the input reconstructor takes advantage of local frequency measurement, $\Delta \omega$, to reconstruct $u$. For load $i \in I$, the estimate of the state vector $x_{t}$ using frequency measurements up to time $(t-1)$ is denoted by $\hat{x}_{t \mid t-1}^{i}$, the estimate of $x_{t}$ using frequency measurements up to time $t$ is denoted by $\hat{x}_{t \mid t}^{i}$, and the estimate of $u_{t-1}$ is denoted by $\hat{u}_{t-1}^{i}$. In the input reconstructor, $\hat{x}_{t \mid t-1}^{i}, \hat{x}_{t \mid t}^{i}$ and $\hat{u}_{t-1}^{i}$ are recursively given by

$$
\begin{gathered}
\hat{x}_{t \mid t-1}^{i}=A \hat{x}_{t-1 \mid t-1}^{i}, \\
\hat{u}_{t-1}^{i}=(C B)^{-1}\left(\Delta \omega_{t}-C \hat{x}_{t \mid t-1}^{i}\right), \\
\hat{x}_{t \mid t}^{i}=\hat{x}_{t \mid t-1}^{i}+B \hat{u}_{t-1}^{i} .
\end{gathered}
$$

The input reconstructor presented by (9)-(11) is a special case of the unbiased minimum-variance input estimator in [24] when process noise and measurement noise are zero. The following proposition states that the input reconstructor is able to reconstruct the value of $u$ locally at each load.

Proposition 1. Suppose Assumption 1 holds, $\hat{x}_{0 \mid 0}^{i}=x_{0}$. Then, the input reconstructor (9)-(11) gives $\hat{u}_{t-1}^{i}=u_{t-1}$ for all $i \in I$ at all times $t \in T$.

$$
\begin{aligned}
& \text { Proof: If } \hat{x}_{t-1 \mid t-1}^{i}=x_{t-1} \text {, by (1), (9) and (10), } \\
& \quad \hat{u}_{t-1}^{i}=(C B)^{-1}\left(\Delta \omega_{t}-C \hat{x}_{t \mid t-1}^{i}\right) \\
& \quad=(C B)^{-1}\left(C A x_{t-1}+C B u_{t-1}-C A \hat{x}_{t-1 \mid t-1}^{i}\right) \\
& =(C B)^{-1} C B u_{t-1}=u_{t-1} .
\end{aligned}
$$

\footnotetext{
${ }^{1}$ We abuse the notation by referring to $\Delta d_{i}(\cdot)$ as a function of time on the left of the equation and a function of $p$ on the right. The meaning should be clear from the context.
}

By (1), (9), (11) and (12), we have

$$
\begin{aligned}
\hat{x}_{t \mid t}^{i} & =\hat{x}_{t \mid t-1}^{i}+B \hat{u}_{t-1}^{i} \\
& =A \hat{x}_{t-1 \mid t-1}^{i}+B u_{t-1} \\
& =A x_{t-1}+B u_{t-1}=x_{t} .
\end{aligned}
$$

Starting from $\hat{x}_{0 \mid 0}^{i}=x_{0}$ and recursively using (12)-(13), we conclude the proof.

Based on the input reconstructor, we design decentralized load control algorithms.

\section{B. Synchronous algorithm}

In the synchronous algorithm, each user senses the power line frequency without measurement delay, and makes decisions synchronously at times $t \in T=\{1,2, \ldots\}$. The synchronous algorithm forms the basis of the asynchronous algorithm, which will be proposed in Section III-C.

We present the synchronous algorithm as follows.

Algorithm 1. Synchronous frequency-based load control algorithm

For all loads $i \in I=\{1,2, \ldots N\}$, the discrete-time realization of the system process model $M$, denoted by $\{A, B, C\}$, is given. Choose a stepsize $\gamma>0$. Set the time $t=0$, and initialize each load $i$ with $\hat{x}_{0 \mid 0}^{i}=x_{0}$ and $p_{i}(0)=0$.

At times $t=1,2, \ldots$, load $i$ :

1) Measures the frequency deviation $\Delta \omega_{t}$, and calculates $\hat{u}_{t-1}^{i}$ using (9)-(11).

2) Updates the value of $p_{i}$ as

$$
p_{i}(t)=p_{i}(t-1)+\gamma \hat{u}_{t-1}^{i} .
$$

3) Determines the change in its load

$$
\Delta d_{i}(t)=\Delta d_{i}\left(p_{i}(t)\right),
$$

where $\Delta d_{i}(\cdot)$ is given by (5).

We make some remarks on the initialization and stop criteria of the algorithm. For the initialization, provided all the eigenvalues of the matrix $A_{c}$ in the continuous-time system realization $\left\{A_{c}, B_{c}, C_{c}\right\}$ (see (2)) have negative real parts, the state vector $x$ converges to the zero vector when power demand and supply are balanced. Therefore, we can set $t=0$ when the system has operated with balanced demand and supply for a relatively long period, and set $\hat{x}_{0 \mid 0}^{i}=0$ for all $i$. Numerical experiments show that the algorithm still converges even if $\hat{x}_{0 \mid 0}^{i} \neq x_{0}$. For the stop criteria, the algorithm can run all the time without stop until when the system model changes, or it can be stopped when $\left|\Delta d_{i}(t+1)-\Delta d_{i}(t)\right|<\epsilon$ for some $\epsilon>0$ for all $i \in I$.

To show the convergence of Algorithm 1, we relate it to the gradient algorithm where the dual variable $p$ is adjusted by (8). Starting with $p(0)=0$, the gradient algorithm produces a sequence $\{p(t), t \in T\}$. Using Algorithm 1, load $i \in I$ generates a sequence $\left\{p_{i}(t), t \in T\right\}$. By (8), (14) and Proposition $1, p_{i}(t)=p(t)$ for all $t \in T$, which implies Algorithm 1 is equivalent to the gradient algorithm if the 
conditions for Proposition 1 hold. Moreover, if Assumptions 23 are satisfied, the dual objective function $U$ has the properties which gurantee the convergence of the gradient algorithm. Hence Assumptions 1-3 together lead to the convergence of Algorithm 1. Define $\bar{\alpha}:=\max _{i \in I} \bar{\alpha}_{i}$ (see $\bar{\alpha}_{i}$ in Assumption 3 ), and define $\Delta d(t):=\left[\Delta d_{1}(t), \ldots, \Delta d_{N}(t)\right]^{T}$. The following theorem states the convergence of Algorithm 1.

Theorem 1. Suppose Assumptions 1-3 hold and the stepsize $\gamma$ satisfies

$$
0<\gamma<\frac{2}{\bar{\alpha} N}
$$

then for any $i \in I$, any limit point (at least one exists) of the sequence $\left\{\left(\Delta d(t), p_{i}(t)\right), t \in T\right\}$ is primal-dual optimal for problems $\mathbf{P P}$ and $\mathbf{D P}$.

Proof: See Appendix A.

We now discuss the convergence rate when $p^{*}$ is the unique maximizer of the dual objective function $U$. We omit the subscript $i$ denoting load $i$ since the sequence $\left\{p_{i}(t), t \in T\right\}$ is the same for all $i$. It is straightforward that

$$
p(t+1)-p^{*}=\left(1+\gamma U^{\prime \prime}(\tilde{p}(t))\right)\left(p(t)-p^{*}\right),
$$

for some $\tilde{p}(t)$ between $p(t)$ and $p^{*}$. Note that $U^{\prime \prime}(\tilde{p}(t))<$ 0 since $U$ is concave and has a unique maximizer, and $U^{\prime \prime}(\tilde{p}(t))>-2$ by the convergence condition (16). Therefore, $\left|1+\gamma U^{\prime \prime}(\tilde{p}(t))\right|<1$ always holds. In addition, near $p^{*}$, the $p(t)$ converges to $p^{*}$ as $t \rightarrow+\infty$ almost as fast as a geometric series with an exponent $\left(1+\gamma U^{\prime \prime}\left(p^{*}\right)\right)$.

Algorithm 1 assumes an ideal case that all loads sense the frequency without measurement delay and make decisions on the change in their power synchronously at every time $t \in T$. However, this may not be true in practice. Now we come to the asynchronous case, where the frequency measurement may be delayed for a random but bounded time, and not all loads make decisions simultaneously.

\section{Asynchronous algorithm}

In the asynchronous setting, the frequency deviation $\Delta \omega_{t}$ at time $t$ is measured by load $i \in I$ at some time within the interval $[t+r(i, t)-1, t+r(i, t))$, where $r(i, t) \in \mathbb{N}$ is a random number. In the time interval $[t-1, t)$, load $i$ measures a set of frequency deviation signals, denoted by $\Omega_{i, t}=\left\{\Delta \hat{\omega}_{i, t}^{1}, \ldots, \Delta \hat{\omega}_{i, t}^{K_{i, t}}\right\}$, where $K_{i, t}$ is the number of measured frequency deviation signals $\left(\Omega_{i, t}=\emptyset\right.$ and $K_{i, t}=0$ means no frequency deviation signal is measured during $[t-1, t))$. Moreover, load $i$ is able to change its power only at a subset of the times, denoted by $T_{i} \subseteq T$. For the asynchronous algorithm to converge, we make the following two assumptions on $r(i, t)$ and $T_{i}$.

Assumption 4. For all $i \in I, t \in T, l \in\left\{1, \ldots, K_{i, t}\right\}$ and $s \in$ $\{1, \ldots, t\}$, if $\Delta \hat{\omega}_{i, t}^{l}$ is the measurement of $\Delta \omega_{s}$, then $\Delta \hat{\omega}_{i, t}^{l+1}$ is the measurement of $\Delta \omega_{s+1}$. Moreover, there exists $\bar{r} \in \mathbb{N}$ such that $r(i, t) \leq \bar{r}$ for all $i \in I$ and $t \in T$.

Assumption 5. For all $i \in I$, the difference between consecutive elements of $T_{i}$ is bounded.
Assumption 4 says that the delayed frequency measurements arrive by order. In other words, the frequency deviation signal that occurs first is sensed first by the load. Moreover, all frequency measurement delays are bounded by $\bar{r}$. Assumption 5 says that the time between any consecutive changes of any load is bounded.

With the setting above, we present the asynchronous load control algorithm as follows.

Algorithm 2. Asynchronous frequency-based load control algorithm

For all loads $i \in I=\{1,2, \ldots N\}$, the discrete-time realization of the system process model $M$, denoted by $\{A, B, C\}$, is given. Choose a stepsize $\gamma>0$. Set the time $t=0$, and initialize each load $i$ with $\hat{x}_{0}^{i}=x_{0}$ and $p_{i}(0)=0$.

In the time interval $[t-1, t)$ for $t=1,2, \ldots$, load $i$ :

1) At time $(t-1)$, sets $\hat{x}_{t-1}^{i}(0 \mid 0)=\hat{x}_{t-1}^{i}, p_{t-1}^{i}(0)=p_{i}(t-$ 1).

2) Once load $i$ measures a new frequency deviation signal $\Delta \hat{\omega}_{i, t}^{k} \in \Omega_{i, t}$ for $k=1, \ldots, K_{i, t}$, it calculates $\hat{u}_{t-1}^{i}(k)$ by

$$
\begin{gathered}
\hat{x}_{t-1}^{i}(k \mid k-1)=A \hat{x}_{t-1}^{i}(k-1 \mid k-1) \\
\hat{u}_{t-1}^{i}(k)=(C B)^{-1}\left(\Delta \hat{\omega}_{i, t}^{k}-C \hat{x}_{t-1}^{i}(k \mid k-1)\right) \\
\hat{x}_{t-1}^{i}(k \mid k)=\hat{x}_{t-1}^{i}(k \mid k-1)+B \hat{u}_{t-1}^{i}(k),
\end{gathered}
$$

and updates the value of $p$ by

$$
p_{t-1}^{i}(k)=p_{t-1}^{i}(k-1)+\gamma \hat{u}_{t-1}^{i}(k) .
$$

3) At time $t$, sets $\hat{x}_{t}^{i}=\hat{x}_{t-1}^{i}\left(K_{i, t} \mid K_{i, t}\right)$ and $p_{i}(t)=$ $p_{t-1}^{i}\left(K_{i, t}\right)$.

4) If load $i$ is able to change its power at time $t$, it determines the change

$$
\Delta d_{i}(t)=\Delta d_{i}\left(p_{i}(t)\right) .
$$

Otherwise, $\Delta d_{i}(t)=\Delta d_{i}(t-1)$.

The following theorem states the convergence of Algorithm 2.

Theorem 2. Suppose Assumptions 1-5 hold and the stepsize $\gamma$ satisfies

$$
0<\gamma<\frac{1}{\bar{\alpha} N / 2+2 \bar{r}},
$$

then for any $i \in I$, any limit point (at least one exists) of the sequence $\left\{\left(\Delta d(t), p_{i}(t)\right), t \in T\right\}$ is primal-dual optimal for problems $\mathbf{P P}$ and $\mathbf{D P}$.

Proof: See Appendix B.

The proposed load control algorithms are essentially the gradient algorithm, where the gradient of the dual objective function happens to be the system demand-supply imbalance $u$. Using the input reconstructor, the loads reconstruct the value of $u$ purely from local frequency measurements, without any communication between the loads and a central controller. 


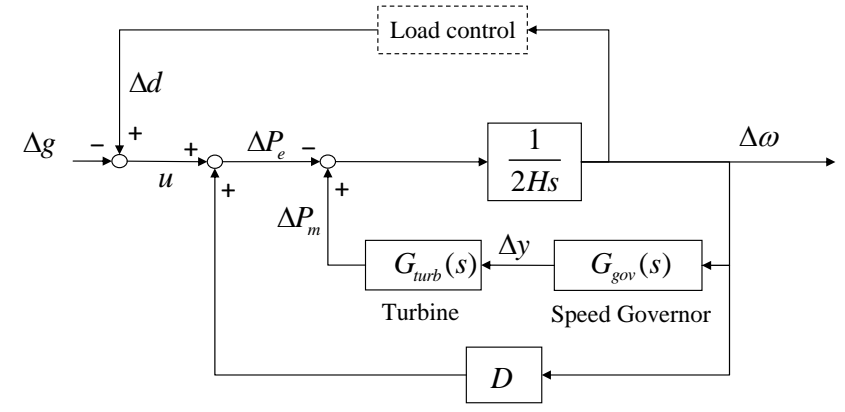

Fig. 2. Core part of the power system model, denoted by $M_{1} . H$ is the generator inertia constant. $D$ is the damping constant of motor loads. $\Delta P_{e}$ in the change in electrical power. The speed governor adjusts the change in turbine valve opening $\Delta y$ based on the frequency deviation $\Delta \omega$, then the change in mechanical power $\Delta P_{m}$ is adjusted by the turbine.

Hence, no communication delay is involved in the proposed scheme. Moreover, the loads sense the local frequency signals with relatively small measurement delays, and respond within a short period of time. Therefore, the proposed load control scheme is able to relatively quickly balancing the demand and supply and restoring the frequency.

\section{CASE STUdies}

In this section, we use relatively detailed power system models to evaluate the performance of the proposed load control scheme. Through numerical experiments with generationloss like contingencies, we compare between the synchronous algorithm and the asynchronous algorithm, show the impact of different numbers of loads, and discuss the effect of using a simplified system model instead of an accurate model. Since the load control under renewable generation is one of our goals to extend, we also test the proposed scheme with a fluctuating wind power generation, though no theoretical result about its performance has been proved for this case.

\section{A. System settings}

We now present the power system model under consideration. Figure 2 shows the core part of the model, denoted by $M_{1}$, which contains the swing equation, the frequency sensitivity of motor loads and the speed governor. The components in $M_{1}$ are major factors that characterize the frequency response caused by demand-supply imbalance [1][7], so we call $M_{1}$ as the core part. In $M_{1}$, the motion of generator rotor is characterized by the swing equation

$$
2 H \frac{d \Delta \omega}{d t}=\Delta P_{m}-\Delta P_{e}
$$

where $H$ is the inertia constant, $\Delta P_{m}$ is the change in mechanical power and $\Delta P_{e}$ is the change in electrical power. For loads equipped with motors, the power is frequency sensitive, described by

$$
\Delta P_{e}(\Delta \omega)=D \Delta \omega
$$

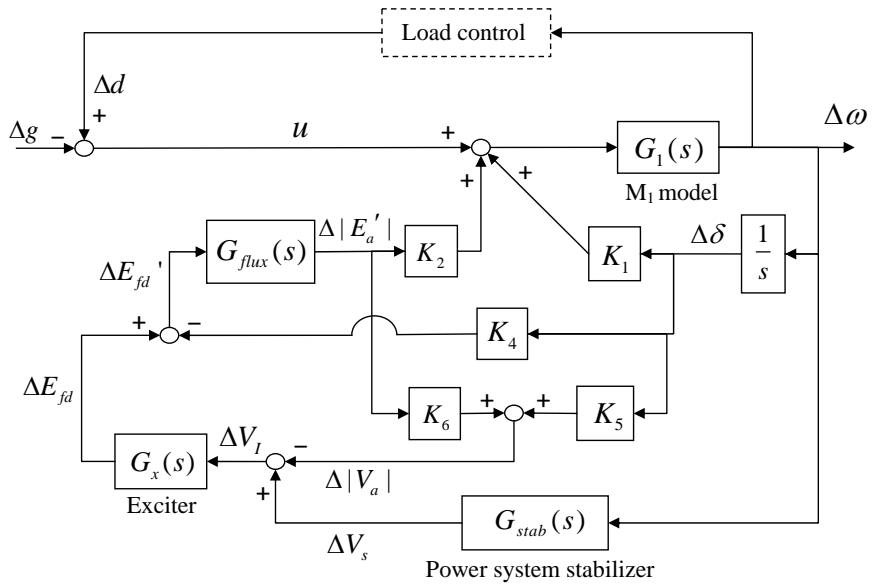

Fig. 3. Complete power system model under consideration, denoted by $M_{2}$. $M_{2}$ contains $M_{1}$. Besides, the exciter adjusts the change in field-winding voltage $\Delta E_{f d}$ based on the change in terminal voltage magnitude $\Delta\left|V_{a}\right|$ and a signal $\Delta V_{s}$ from the power system stabilizer. $\Delta \delta$ is the change in rotor angle and $\Delta\left|E_{a}^{\prime}\right|$ is the change in stator voltage magnitude.

where $D$ is the damping constant. Moreover, the model contains a speed governor with the transfer function

$$
G_{\text {gov }}(s)=-\frac{1}{R\left(1+s T_{G}\right)}
$$

such that the change in the turbine valve opening $\Delta y(s)=$ $G_{\text {gov }}(s) \Delta \omega(s)$. The turbine has a transfer function

$$
G_{\text {turb }}(s)=\frac{\left(1+s F_{H P} T_{R H}\right)}{\left(1+s T_{C H}\right)\left(1+s T_{R H}\right)}
$$

such that $\Delta P_{m}(s)=G_{\text {turb }}(s) \Delta y(s)$. Hence, the transfer function of $M_{1}$, denoted by $G_{1}$, is

$$
G_{1}(s)=-\frac{1 /(2 H s+D)}{1-1 /(2 H s+D) G_{\text {turb }}(s) G_{\text {gov }}(s)} .
$$

The complete model under consideration, denoted by $M_{2}$, is shown in Figure 3. Besides the core part $M_{1}, M_{2}$ contains a power system stabilizer (PSS) with the transfer function

$$
G_{s t a b}(s)=\frac{s K_{w}\left(1+s T_{1}\right)\left(1+s T_{3}\right)}{\left(1+s T_{w}\right)\left(1+s T_{2}\right)\left(1+s T_{4}\right)},
$$

such that a voltage signal $\Delta V_{s}(s)=G_{s t a b}(s) \Delta \omega(s)$ is delivered to the exciter. The exciter also receives the change in magnitude of the terminal voltage $\Delta\left|V_{a}\right|$. Here we use an IEEE AC4A exciter, which has the transfer function

$$
G_{x}(s)=\frac{K_{A}\left(1+s T_{C}\right)}{\left(1+s T_{A}\right)\left(1+s T_{B}\right)}
$$

that characterizes the relation between the change in fieldwinding voltage $\Delta E_{f d}$ and the change in exciter input voltage $\Delta V_{I}$. Moreover, the generator has a flux decay transfer function

$$
G_{f l u x}(s)=\frac{K_{3}}{1+K_{3} \tau_{d 0}^{\prime} s}
$$

such that the change in stator voltage magnitude $\Delta\left|E_{a}^{\prime}\right|(s)=$ $G_{f l u x}(s) \Delta E_{f d}^{\prime}(s)$, where $\Delta E_{f d}^{\prime}=\Delta E_{f d}-K_{4} \Delta \delta$, i.e., the 
TABLE I

PARAMETERS USED IN THE CASE STUDIES

\begin{tabular}{|c|c||c|c||c|c|}
\hline Param. & Value & Param. & Value (s) & Param. & Value $(\mathrm{s})$ \\
\hline$K_{A}$ & 200 & $H$ & 5 & $T_{1}$ & 0.2 \\
$K_{1}$ & 1.0755 & $T_{A}$ & 0.04 & $T_{2}$ & 0.02 \\
$K_{2}$ & 1.2578 & $T_{B}$ & 12 & $T_{3}$ & 0.4 \\
$K_{3}$ & 0.3072 & $T_{C}$ & 1 & $T_{4}$ & 0.04 \\
$K_{4}$ & 1.7124 & $\tau_{d 0}^{\prime}$ & 5.9 & $T_{w}$ & 10 \\
\hline Param. & Value & Param. & Value $(\mathrm{s})$ & Param. & Value $(\mathrm{pu})$ \\
\hline$K_{5}$ & -0.0409 & $T_{G}$ & 0.2 & $D$ & 1 \\
$K_{6}$ & 0.4971 & $T_{C H}$ & 0.3 & $R$ & 0.05 \\
$K_{w}$ & 20 & $T_{R H}$ & 7 & $F_{H P}$ & 0.3 \\
\hline
\end{tabular}

rotor angle $\Delta \delta$ has a negative feedback to the field-winding voltage. We have the transfer function of $M_{2}$, denoted by $G_{2}$, as

$$
G_{2}(s)=\frac{G_{1}(s)}{1-G_{1}(s)\left[F(s)+K_{1} / s\right]},
$$

where $G_{1}(s)$ is given in (18) and $F(s)$ is (omitting the $(s)$ following the transfer functions and variables for simplicity)

$$
\begin{aligned}
& F=\frac{K_{2} \Delta\left|E_{a}^{\prime}\right|}{\Delta \omega} \\
& =\frac{K_{2} G_{\text {flux }}\left(-K_{5} G_{x} / s-K_{4} / s+G_{x} G_{\text {stab }}\right)}{1+K_{6} G_{\text {flux }} G_{x}} .
\end{aligned}
$$

The system under consideration is a per unit system with baseline power $P_{\text {base }}=2000$ MVA. The sampling time is $\Delta t=0.1 \mathrm{~s}$. There are $N$ loads in each experiment. Load $i \in I=\{1,2, \ldots, N\}$ has a disutility function $D_{i}\left(\Delta d_{i}\right)=(1 / 2) \bar{\alpha}_{i} \Delta d_{i}^{2}$, where $\bar{\alpha}_{i}$ is a random number, e.g., uniformly distributed on $[1,3]$ in our experiments. For $i \in I$, $\Delta d_{i} \in\left[0, \bar{d}_{i}\right]$, where $\bar{d}_{i}$ is a positive random number and $\sum_{i \in I} \bar{d}_{i}=0.30$ per unit (pu). In the asynchronous setting, the upper bound for all frequency measurement delays is $\bar{r}=2$ (see Assumption 4 for $\bar{r}$ ), and the set of decision times of load $i$ is $T_{i}=T=\{1,2,3, \ldots\}$ for $i \in\{1, \ldots, N / 4\}$, $T_{i}=\{5,10,15, \ldots\}$ for $i \in\{N / 4+1, \ldots, N / 2\}, T_{i}=$ $\{10,20,30, \ldots\}$ for $i \in\{N / 2+1, \ldots, 3 N / 4\}$, and $T_{i}=$ $\{50,100,150, \ldots\}$ for $i \in\{3 N / 4+1, \ldots, N\}$. All loads run the load control algorithm with the stepsize $\gamma=0.9 /(\bar{\alpha} N / 2+2 \bar{r})$, where $\bar{\alpha}=\max _{i \in I} \bar{\alpha}_{i}$. Therefore, all the sufficient conditions for the convergence of Algorithms 1 and 2 are satisfied. Table I gives the other parameters values used in the case studies.

\section{B. Generation-loss like contingencies}

We use the disturbance $\Delta g$ which contains two step changes to resemble generation-loss like contingencies, and observe the performance of the proposed load control scheme. $\Delta g$ is initially zero. At time $t=2 \mathrm{~s}$, the first step change occurs with $\Delta g$ falling by $0.05 \mathrm{pu}$; at $t=20 \mathrm{~s}$, the second step change occurs with $\Delta g$ falling further by $0.20 \mathrm{pu}$.

Figure 4 shows the frequency response of the complete system model $M_{2}$ to the step changing disturbance, with the synchronous or the asynchronous algorithm. Both algorithms improve the frequency response with small deviations from

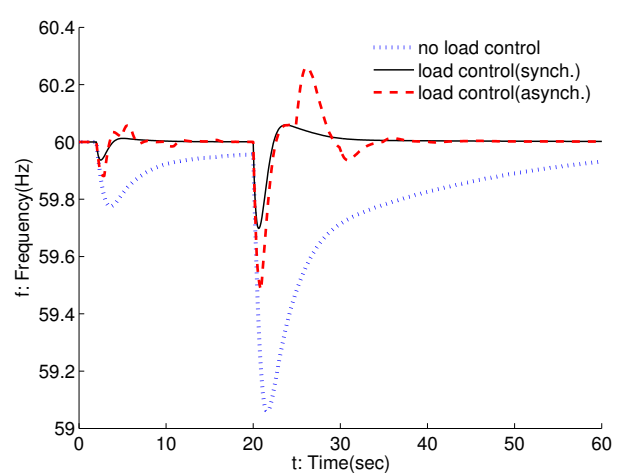

Fig. 4. Frequency reponse with the synchronous or the asynchronous algorithm. The dotted line is the frequency response without load control. The solid line and the dashed line are respectively the frequency response with load control using the synchronous and the asynchronous algorithm.

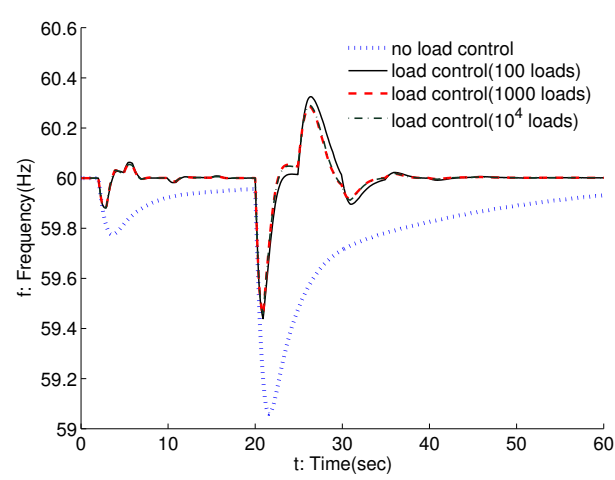

Fig. 5. Frequency reponse with different numbers of loads. The dotted line is the frequency response without load control. The solid line, the dashed line and the dash-dot line are respectively the frequency response with load control algorithms applied on 100, 1,000 and 10,000 loads.

and quick convergence (within $10 \mathrm{~s}$ after the first generation fall and $20 \mathrm{~s}$ after the second) to $60 \mathrm{~Hz}$. The synchronous algorithm performs better with even smaller oscillations in transient frequency response than the asynchronous algorithm, because in the synchronous algorithm all the loads are adjusted to the optimal power given the newest gradient value simultaneously at every sampling time, while in the asynchronous algorithm loads get delayed gradient value and some loads are not adjusted at some times.

Figure 5 shows the frequency response of the complete system model $M_{2}$ to the step changing disturbance, with the asynchronous algorithm applied on different numbers of loads. We see that the number of loads does not affect the frequency response very much. As the number of loads increases, the proposed load control scheme maintains its response speed and convergence rate as there is a small number of loads. In other words, decentralization to more loads does not compromise the performance of the proposed load control scheme.

Now we discuss the effect of using a simplified model instead of an accurate model in the load control scheme. In both Algorithms 1 and 2, a discrete-time realization of 


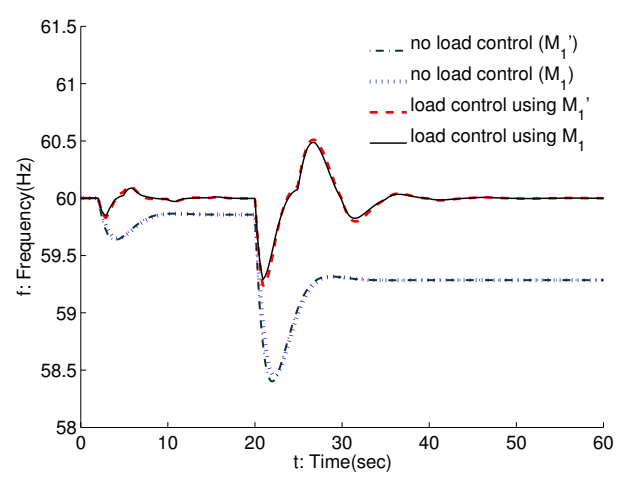

Fig. 6. Frequency reponse of the core part model $M_{1}$ and the simplified model $M_{1}^{\prime}$. The dotted line and the dash-dot line are respectively the frequency response of $M_{1}$ and $M_{1}^{\prime}$ without load control. The solid line and the dashed line are respectively the frequency response of $M_{1}$ with load control using $M_{1}$ and using $M_{1}^{\prime}$.

the system model, denoted by $\{A, B, C\}$, is given to every load. However, as is mentioned in Section I, users may not get an accurate model, since the utility company may not reveal the exact system information to the public for security considerations, and the accurate model may be so complicated that it costs much to determine it, deliver it to users and compute with it. Hence, we consider the effect of using a simplified model in the load control algorithm.

For the complete system model $M_{2}$, we derive its simplifed model from its core part $M_{1}$, since $M_{1}$ captures the main characteristics of the frequency response. Note that the transfer function of $M_{1}$, denoted by $G_{1}$, has a pair of conjugate complex poles which are close to the imaginary axis for the given parameter values. With this condition, we simplify $G_{1}$ by discarding poles and zeros that are far from the imaginary axis and canceling zero-pole pairs that are close to each other [28], and have a simplified transfer function

$$
\widetilde{G}_{1}(s)=-\frac{0.1555 s+0.0222}{s^{2}+0.9918 s+0.4666} .
$$

which describes a simplified model $M_{1}^{\prime}$. Figure 6 compares the frequency response of $M_{1}$ and $M_{1}^{\prime}$. As we can see, $M_{1}^{\prime}$ approximates $M_{1}$ very well, since its frequency response curve without load control almost overlaps with that of $M_{1}$, and the frequency response curves with load control using $M_{1}$ and $M_{1}^{\prime}$ are quite similar. Hence, $M_{1}^{\prime}$ can be regarded as a simplification of the core part model $M_{1}$, and a simplification of the complete model $M_{2}$.

Figures 7-9 respectively compare the frequency response, the total change in load and the disutility with load control schemes using the complete model $M_{2}$ and the simplified model $M_{1}^{\prime}$. Comparing the no-load-control frequency response curves of $M_{2}$ (the dotted line) and $M_{1}^{\prime}$ (the dashdot line) in Figure 7, we see that $M_{1}^{\prime}$ is not an accurate simplification of $M_{2}$. However, the frequency response, the total change in load and the disutility with load control schemes using $M_{2}$ and $M_{1}^{\prime}$ are still quite close (though there are small steady state differences after the second step change

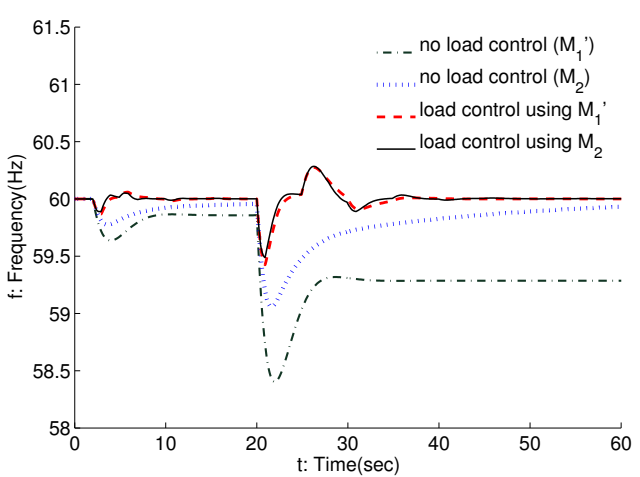

Fig. 7. Frequency reponse with load control schemes using the complete model $M_{2}$ and the simplified model $M_{1}^{\prime}$. The dotted line and the dash-dot line are respectively the frequency response of $M_{2}$ and $M_{1}^{\prime}$ without load control. The solid line and the dashed line are respectively the frequency response with load control schemes using $M_{2}$ and using $M_{1}^{\prime}$.

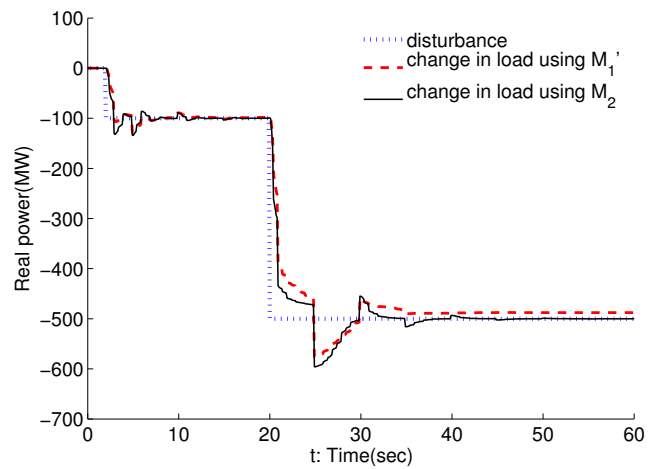

Fig. 8. Total change in load with load control schemes using the complete model $M_{2}$ and the simplified model $M_{1}^{\prime}$. The dotted line is the disturbance $\Delta g$. The solid line and the dashed line are respectively the total change in load with load control schemes using $M_{2}$ and using $M_{1}^{\prime}$.

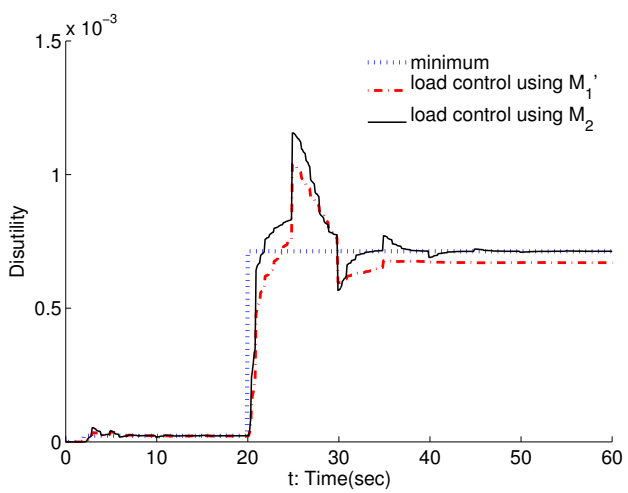

Fig. 9. Disutility caused by load control schemes using the complete model $M_{2}$ and the simplified model $M_{1}^{\prime}$. The dotted line is the minimal disutility that can be achieved. The solid line and the dashed line are respectively the disutility caused by load control schemes using $M_{2}$ and using $M_{1}^{\prime}$. 


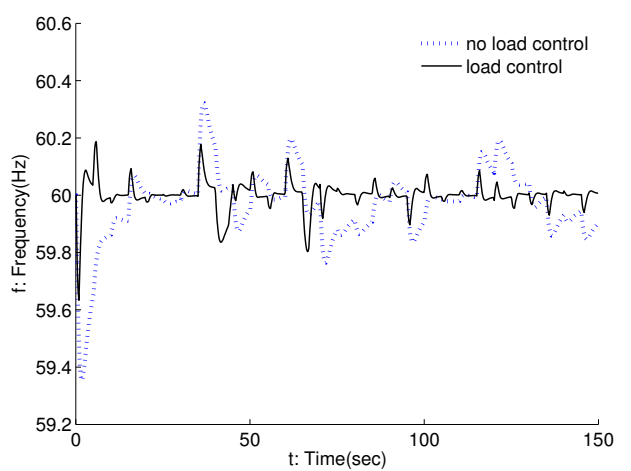

Fig. 10. Frequency reponse to wind power injection. The dotted line and the solid line are respectively the frequency response without load control and with the load control scheme using the asynchronous algorithm.

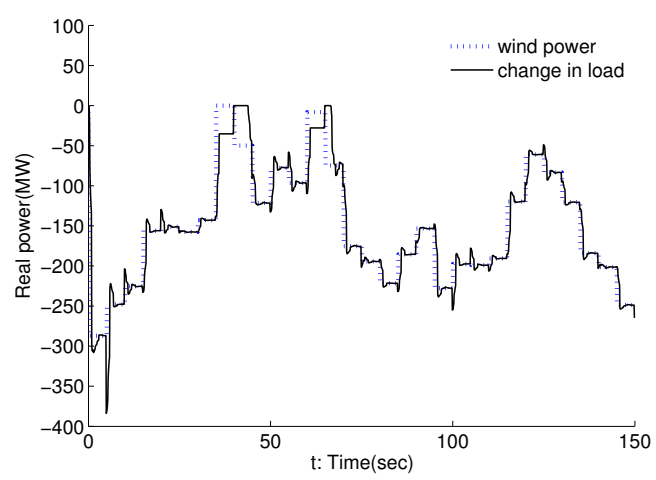

Fig. 11. Total change in load under wind power injection. The dotted line is the wind power generation. The solid line is the total change in load with the load control scheme using the asynchronous algorithm.

in Figures 8 and 9), which implies the proposed scheme is robust to inaccurate model simplifications. Therefore, it is tolerable to use a simplified system model, instead of an accurate one, in the load control scheme.

\section{Wind power generation}

Now we show the performance of the proposed load control scheme when power generated by a wind turbine is injected to the system. Though we have not theoretically proved any result about the proposed scheme with time-varying disturbance $\Delta g$, we test with the wind power generation to see whether the proposed load control scheme does any better than that without load control, as a prelude to studying detailed algorithms serving the control purposes under renewable generations.

The $\Delta g$ trace, which resembles the wind power generation, comes from actual wind turbine power output data [11]. $\Delta g$ varies every 5 seconds. Figures 10 and 11 respectively show the frequency response and the total change in load with the load control scheme using the asynchronous algorithm. Figure 10 shows that with the proposed load control scheme the frequency deviation from $60 \mathrm{~Hz}$ becomes smaller than that without load control for most of the time. In addition, the largest absolute value of frequency deviation is $0.6463 \mathrm{~Hz}$ without load control and $0.3517 \mathrm{~Hz}$ with the proposed load control scheme, nearly $54 \%$ of the former. The root of mean square frequency deviation is $0.1348 \mathrm{~Hz}$ without load control and $0.0513 \mathrm{~Hz}$ with the proposed load control scheme, nearly $38 \%$ of the former. Figure 11 shows that with the proposed load control scheme, the total change in load follows the wind power generation quite well. Experiments with other traces of wind power generation show similar results. At least for the cases we test, the proposed load control scheme does better than that without load control in regulating frequency and balancing demand with supply under wind power generation.

\section{LiMITATIONS AND EXTENTIONS}

The proposed load control scheme has several limitations. First, we characterize the frequency response to demandsupply power imbalance and derive the load control algorithms based on a linearized power system model, which is suitable for small deviations from the operating point but produces large error when the deviations grow large. Second, we do not consider the model error and the process and measurement noise, which may be a significant issue in practice. Third, we only study a simple power system with a single generator and all the loads sensing the same power line frequency, but have not considered the power network composed of multiple interconnected buses at each the frequency may be different.

These limitations motivate some possible extensions based on our work. In the future, we will work on the robustness issue, i.e., study the performance of the frequency-based load control scheme with model error and noise. Moreover, we will study with more detailed and realistic power system model which may be non-linear and contain multiple synchronous machines and buses so that loads at different sites may have different frequency measurements. In that case, some communication may be required to coordinate the actions of different groups of loads. With these considerations the load control scheme design would become more interesting and practical.

\section{CONCLUSION}

In this paper, we present a frequency-based load control scheme to balance demand with supply and regulate frequency in power systems. We set up a load control optimization problem with the objective of minimizing the overall end-use disutility and the constraint of demand-supply power balance. We design decentralized algorithms using local frequency measurements to solve the load control optimization problem, and prove the convergence of the algorithms. Numerical experiments show that the proposed load control scheme is able to relatively quickly balance the power demand with supply and restore frequency under generation-loss like contingencies or renewable power generation. In addition, the proposed scheme still has good performance when an inaccurate, simplified system model, instead of an accurate one, is used in the load control algorithm. 


\section{APPENDIX A \\ PROOF OF THEOREM 1}

To prove Theorem 1 (convergence of Algorithm 1), we first give two lemmas that show the properties of the dual objective function $U$. We skip their proofs since the proof of Lemma 1 follows directly from Assumption 2 and the proof of Lemma 2 uses the similar way with the proof of [19, Lemma 2-3].

Lemma 1. Suppose Assumption 2 holds. Then the dual objective function $U$ is concave, continuously differentiable and bounded above over $\mathbb{R}$.

Lemma 2. Suppose Assumptions 2-3 hold. Then for any $p, q \in \mathbb{R}$

$$
\left|U^{\prime}(q)-U^{\prime}(p)\right| \leq \bar{\alpha} N|q-p|,
$$

where $\bar{\alpha}:=\max _{i \in I} \bar{\alpha}_{i}$.

Now we complete the proof of Theorem 1. Let $\{p(t), t \in T\}$ denote the sequence generated by the gradient algorithm, and $\left\{p_{i}(t), t \in T\right\}$ denote the sequence generated by load $i \in I$ using Algorithm 1. Recall that by Proposition 1, $p_{i}(t)=p(t)$ for all $i \in I$ and all $t \in T$. By Lemma 1-2, the dual objective function $U$ is concave and bounded above, and $U^{\prime}$ is Lipschitz continuous over $\mathbb{R}$. Following [20, pp. 203-204], if $0<\gamma<$ $2 /(\bar{\alpha} N)$, then

$$
\lim _{t \rightarrow \infty} U^{\prime}\left(p_{i}(t)\right)=0 .
$$

Let $p^{*}$ denote any limit point of $\left\{p_{i}(t), t \in T\right\}$, then $U^{\prime}\left(p^{*}\right)=$ 0 . Since $U$ is concave over $\mathbb{R}$ and the dual problem DP is unconstrained, $p^{*}$ is a maximizer of DP. It is easy to see that the level set $\left\{p \in \mathbb{R} \mid U(p) \geq U\left(p_{i}(0)\right)\right\}$ is compact. Moreover, $U\left(p_{i}(t)\right)$ is non-decreasing [20, pp. 204]. Thus $p_{i}(t)$ is always in a compact set for all $t$. By the definition of compactness, there exists at least one limit point of $\left\{p_{i}(t), t \in T\right\}$, denoted by $p^{*}$. By (5), $\Delta d_{i}(\cdot)$ is continuous over $\mathbb{R}$, thus $\Delta d_{i}\left(p^{*}\right)$ is a limit point of $\left\{\Delta d_{i}(t), t \in T\right\}$. By strong duality between $\mathbf{P P}$ and DP $, \Delta d\left(p^{*}\right):=\left[\Delta d_{1}\left(p^{*}\right), \ldots, \Delta d_{N}\left(p^{*}\right)\right]^{T}$ is primal optimal since $p^{*}$ is dual optimal (see Section II-B).

\section{APPENDIX B \\ PROOF OF THEOREM 2}

To prove Theorem 2 (convergence of Algorithm 2), we first show that Algorithm 2 is a special case of the asynchronous flow control algorithm [19, Algorithm A2].

In Algorithm 2, load $i \in I$ generates a sequence

$$
U_{i, t}:=\hat{u}_{0}^{i}(1), \ldots, \hat{u}_{0}^{i}\left(K_{i, 1}\right), \ldots, \hat{u}_{t-1}^{i}(1), \ldots, \hat{u}_{t-1}^{i}\left(K_{i, t}\right)
$$

and a sequence

$$
P_{i, t}:=p_{0}^{i}(1), \ldots, p_{0}^{i}\left(K_{i, 1}\right), \ldots, p_{t-1}^{i}(1), \ldots, p_{t-1}^{i}\left(K_{i, t}\right) .
$$

We formulate another sequence $\{p(t), t \in T\}$, which starts from $p(0)=0$ and evloves as

$$
p(t)=p(t-1)+\gamma u_{t-1} .
$$

Define $\tau(i, t):=\max \{\tau \in T \mid \tau+r(i, \tau)=t\}$ where $r(i, \tau)$ is load $i$ 's measurement delay of $\Delta \omega_{\tau}$. The following lemma is straightforward from (17), (19) and Proposition 1.

Lemma 3. The sequence $U_{i, t}$ is the same as $u_{0}, \ldots, u_{\tau(i, t)-1}$, and the sequence $P_{i, t}$ is the same as $p(1), \ldots, p(\tau(i, t))$.

Recall that in Algorithm 2, $p_{i}(t)=p_{t-1}^{i}\left(K_{i, t}\right)$. Now we can regarding $p(t), u_{t}, p_{i}(t)$ and $\Delta d_{i}(t)$ respectively as the link price $p_{l}(t)$, the estimate of gradient $\lambda_{l}(t)$, the estimate of link price $\hat{p}^{s}(t)$ and the transmission rate $x_{s}(t)$ in the asynchronous flow control algorithm [19, Algorithm A2]. We can see that Algorithm 2 is a special case of the asynchronous flow control algorithm. By the proof of $[19$, Theorem 2], if Assumption 1-5 hold and the stepsize $\gamma$ satisfies $0<\gamma<1 /((\bar{\alpha} N / 2)+2 \bar{r})$, any limit point (at least one exists) of the sequence $\{(\Delta d(t), p(t)), t \in T\}$ is primal-dual optimal for the problems $\mathbf{P P}$ and $\mathbf{D P}$. If follows that for $i \in I$, any limit point (at least one exists) of the sequence $\left\{\left(\Delta d(t), p_{i}(t)\right), t \in T\right\}$ is primal-dual optimal for the problems $\mathbf{P P}$ and $\mathbf{D P}$.

\section{REFERENCES}

[1] P. M. Anderson and M. Mirheydar, "An adaptive method for setting underfrequency load shedding relays," IEEE Trans. on Power Systems, vol. 7, no. 2, pp. 647-655, 1992.

[2] A. Molina-Garcia, F. Bouffard and D. S. Kirschen, "Decentralized demand-side contribution to primary frequency control," IEEE Trans. on Power Systems, vol. 26, no. 1, pp. 411-419, 2001.

[3] D. S. Callaway and I. A. Hiskens, "Achieving controllability of electric loads," Proceedings of the IEEE, vol. 99, no. 1, pp. 184-199, 2011.

[4] M. Ilic and Q. Liu, "Toward sensing, communications and control architectures for frequency regulation in systems with highly variable resources," Control and Optimization Theory for Electric Smart Grids, New York: Springer, 2011.

[5] D. Trudnowski, M. Donnelly and E. Lightner, "Power-system frequency and stability control using decentralized intelligent loads," in Proc. of the 2005 IEEE T\&D Conf. Expo., Dallas, TX, May 2006.

[6] D. Trudnowski, M. Donnelly and E. Lightner, "Power-system frequency and stability control using decentralized intelligent loads: Benefits and pitfalls," in Proc. of the 2010 IEEE Power and Energy Society General Meeting, Minneapolis, MN, Jul. 2010.

[7] J. Dong, J. Zuo, L. Wang, K. S. Kook, I. Chung, Y. Liu, et al., "Analysis of power system disturbance based on wide-area frequency measurements," in Proc. of the 2007 IEEE Power and Energy Society General Meeting, Tampa, FL, Jun. 2007.

[8] P. Kundur, Power System Stability and Control, New York: McGraw Hill, Inc., 1994.

[9] G. Rogers, Power System Oscillations. Boston, MA: Kluwer Academic Publishers, 2000

[10] G. Strbac and ILEX Energy Consulting, Quantifying the System Costs of Additional Renewables in 2020. Department of Trade and Industry, London, U.K., 2002.

[11] Z. Xu, J. Ostergaard, M. Togeby, "Demand as frequency controlled reserve," IEEE Trans. on Power Systems, vol. 26, no. 3, pp. 1062-1071, 2011.

[12] V. N. Chuvychin, N. S. Gurov, S. S. Venkata and R. E. Brown, "An adaptive approach to load shedding and spinning reserve control during underfrequency conditions," IEEE Trans. on Power Systems, vol. 11, no. 4, pp. 1805-1810, 1996.

[13] A. Brooks, E. Liu, D. Reicher, C. Spirakis and B. Weihl, "Demand dispatch: Using real-time control of demand to help balance generation and load," IEEE Power\&Energy Magazine, vol. 8, no. 3, pp. 21-30, 2010.

[14] F. C. Schweppe, "Frequency Adaptive Power-Energy Re-Scheduler," United States Patent 4317049, 1979.

[15] F. C. Schweppe, R. D. Tabors, J. L. Kirtley, H. R. Outhred, F. H. Pickel and A. J. Cox, "Homeostatic utility control," IEEE Trans. Power App. Syst., vol. PAS-99, pp. 1151-1163, May/Jun. 1980. 
[16] P. Taylor, "Increased renewable energy penetration on island power systems through distributed fuzzy load control," in Proc. Int. Conf. Renewable Energies for Islands: Toward 100\% RES Supply, Chania, Greece, Jun. 2001.

[17] K. Pandiaraj, P. Taylor, N. Jenkins and C. Robb, "Distributed load control of autonomous renewable energy systems," IEEE Trans. Energy Conv., vol. 16, no. 1, pp. 14-19, Mar. 2001.

[18] PNNL, Grid Friendly ${ }^{T M}$ Controller Helps Balance Energy Supply and Demand. [Online]. Available: http://www.gridwise.pnl.gov/docs/pnnlsa36565.pdf.

[19] S. H. Low and D. E. Lapsley, "Optimization flow control, I: Basic algorithm and convergence," IEEE/ACM Trans. on Networking, vol. 7, no. 6, pp. 861-874, Dec. 1999.

[20] D. Bertsekas and J. Tsitsiklis, Parallel and Distributed Computation. Upper Saddle River, NJ: Prentice Hall, 1989.

[21] D. Bertsekas, Nonlinear Programming. Nashua, NH: Athena Scientific, 1995.

[22] A. R. Bergen and V. Vittal, Power Systems Analysis, 2nd ed. Upper Saddle River, NJ: Prentice Hall, 2000.

[23] P. M. Anderson and A. A. Fouad, Power System Control and Stability, Volume 1. Ames, Iowa: The Iowa State University Press, 1977.

[24] S. Gillijns and B. D. Moor, "Unbiased minimum-variance input and state estimation for linear discrete-time systems," Automatica, vol. 43, no. 1, pp. 111-116, 2007.

[25] M. Hou and R. J. Patton, "Input observability and input reconstruction," Automatica, vol. 34, no. 6, pp. 789-794, 1998.

[26] S. Boyd and L. Vandenberghe, Convex Optimization, U.K.: Cambridge University Press, 2004

[27] L. Gan, U. Topcu and S. H. Low, "Optimal decentralized protocols for electric vehicle charging," to appear in Proc. 2011 IEEE Conf. Decision and Control, Orlando, FL, Dec. 2011.

[28] K. J. Astrom and R. M. Murray, Feedback Systems: An Introduction for Scientists and Engineers, Princeton, NJ: Princeton University Press, 2008.

[29] W. Rudin, Principles of Mathematical Analysis, 3rd ed. New York: McGraw Hill, Inc., 1976.

[30] N. Li, L. Chen and S. H. Low, "Optimal demand response based on utility maximization in power networks," in Proc. of the 2011 IEEE Power and Energy Society General Meeting, Detroit, MI, Jul. 2011.

[31] P. Samadi, A. Mohsenian-Rad, R. Schober, V. W. S. Wong, and J. Jatskevich, "Optimal real-time pricing algorithm based on utility maximization for smart grid," in Proc. 2010 IEEE SmartGridComm, Gaithersburg, MD, Oct. 2010. 